\title{
Who is More Positive in Private? Analyzing Sentiment Differences across Privacy Levels and Demographic Factors in Facebook Chats and Posts
}

\author{
Bo Gao ${ }^{1}$, Bettina Berendt ${ }^{1}$ and Joaquin Vanschoren ${ }^{2}$ \\ ${ }^{1}$ Dept. Computer Science, KU Leuven. \{bo.gao, bettina.berendt $\} @ c s . k u l e u v e n . b e$ \\ ${ }^{2}$ Dept. Mathematics and Computer Science, TU Eindhoven.j.vanschoren@tue.nl
}

\begin{abstract}
Understanding users' sentiments in social media is important in many domains, such as marketing and online applications. Is one demographic group inherently different from another? Does a group express the same sentiment both in private and public? How can we compare the sentiments of different groups composed of multiple attributes? In this paper, we take an interdisciplinary approach towards mining the patterns of textual sentiments and metadata. First, we look into several existing hypotheses in social science on the interplay between user characteristics and sentiments, as well as the related evidence in the field of social network data analysis. Second, we present a dataset with unique features (Facebook users' chats and posts in multiple languages) and a procedure to process the data. Third, we test our hypotheses on this dataset and interpret the results. Fourth, under the subgroup-discovery paradigm, we present an approach with two algorithms that generalizes single-attribute testing. This approach provides more detailed insight into the relationships among attributes, and reveals interesting attributevalue combinations with distinct sentiments. Furthermore, it offers novel hypotheses for examination in future studies.
\end{abstract}

\section{INTRODUCTION}

Understanding users' sentiments in social media is important in many domains, such as marketing, sociological/psychological study and online application development. For example, in marketing, data analysts monitor and mine texts in social media to discover how participants in specific demographic groups react to certain brands or events. An analyst must be aware of existing sentiment differences. For instance, do older people express themselves more positively? Is there a difference in sentiment expression between married and single people? However, most hypotheses are based on offline studies. It is thus interesting to test and examine them in more detail with online social network data.

Recently, there has been a large interest in Facebook sentiment analysis [1], [2]. To the best of our knowledge, all the existing sentiment analysis has been conducted on status updates, or other (semi-)publicly available data in online social networks. While users post what they think or like publicly, they also chat privately ${ }^{1}$. Is there a sentiment difference between public and private? In this paper, we discover and compare the sentiment patterns in both posts and chats on Facebook

${ }^{1}$ Facebook Chat https://www.facebook.com/help/332952696782239/ in a more differentiated way. Furthermore, most studies have focused on the correlations between singular (demographic) factors and sentiments in online social networks. However, it can be more productive to study the sentiment differences using multiple factors. For example, the male users of 21-24 years old with the "friends" privacy setting (see Section III) are less positive than those of 25-28 year old. This type of pattern mining falls under the Subgroup-Discovery paradigm. We propose two algorithms to extract subgroup comparisons of differentiated sentiments.

The remainder of the paper is organized as follows: in Section II, we explicate our research questions in light of relevant literature; in Section III, we describe our dataset; in Section IV, we test the sentiment differences for singleattribute subgroups; in Section V, we detail our approach to discover "interesting" multi-attribute subgroups; in Section VI, we discuss limitations and outlook; finally, we conclude in Section VII.

\section{RESEARCH QUESTIONS AND RELATED WORK}

We use the term "sentiment" to refer to a simplified attitude or emotional state that can be characterised as positive, negative or neutral. For a given document, the positive sentiment strength is $s^{+}>0$ and its negative sentiment strength is $s^{-}<0$, we consider its expressiveness to be $\left(s^{+}-s^{-}\right)$. We note that the gender-wise online sentiment differences have been extensively studied (e.g. [3]), thus we will not investigate this in single attribute hypothesis testing, but we will see the interactions of "gender" with other attributes in Section V.

When a user posts or chats on Facebook, each post/chat has an audience range, mostly definable by the user. For example, a post's privacy setting can be adjusted from only visible to oneself to the entire web. The number of participants also implicitly defines the audience range of a private chat. Oftentimes, people express themselves positively rather than negatively on Facebook, as negative emotions are not socially favourable and people tend to suppress negative emotions in public [4]. Different levels of privacy settings may trigger different sentiment expressions. Our corresponding research questions are:

RQ1: In Facebook chats and posts, (a) do users express 
more positive and/or less negative sentiment in public than in private? (b) Is there a difference in expressiveness?

For age differentiated emotional behaviour, Gross et al. [5] investigated subjects' emotional experience, expression and control. The results consistently showed that, compared to younger subjects, older subjects reported fewer negative emotional experiences and greater emotional control. Furthermore, Stone et al. [6] found that people's positive emotional state increases after 50 years old. Stress and anger steeply declines from the early 20s. Worry was elevated through middle age (30-59 years old) and then declined. However, do these findings translate to the communication in online social networks? Our corresponding research questions are:

RQ2: In Facebook chats and posts, (a) Does negative sentiment decline after people's early 20s but increase during middle age? (b) Do people older than 50 years old express themselves more positively?

Researchers have also studied emotional differences in terms of relationship status. For instance, Yap et al. [7] found that married people reported higher levels of satisfaction than they did while being single. Another study [8] showed that being in a relationship was associated with higher levels of anger. Our corresponding research questions are:

RQ3: In Facebook chats and posts, (a) do married people express more positive and/or less negative sentiment than single people? (b) Do people in a relationship (not married) express less positive and/or more negative sentiment than single people? (c) Is there a difference between the people who are married and those that are in a relationship?

Finally, besides comparing sentiment differences within single attributes, a more general problem is to discover subgroups of multiple attributes in exploratory data analysis. This falls under the paradigm of subgroup discovery [9]. Our corresponding research question is:

RQ4: How can we discover "interesting" subgroups that help us gain more knowledge with multi-attribute groups?

\section{DATA}

Data Collection and Overview: We built an online visualization tool for Facebook users ${ }^{2}$. With their consent, we collected the egocentric network data of 199 tool users from November, 2013 to January, 2015. The data consists of friend graphs, user profiles, chats and posts. The box plot [11] for the number of friends per user is shown in Figure 1. In total, we identify 66,013 users with profiles, $49.2 \%$ male, $50 \%$ female and unspecified for the rest. $64.6 \%$ of these users specify their birth dates, mostly people in their 20 s. $61 \%$ specified their home towns, out of whom $68 \%$ come from Belgium, the rest are mainly from Spain (5\%), the Netherlands (3\%), Germany $(3 \%)$, Italy (2\%) and France (1\%). In both chats and posts, a user types a main message or a comment to communicate, "text" for short. We use \#texts/user to refer to the distribution of the number of texts sent by a user, \#words/text and \#chars/text to refer to the distributions of the number of words (space-separated) and the number of characters (UTF-8) in a

\footnotetext{
${ }^{2}$ We sent to friends and colleagues the tool link http://people.cs.kuleuven. be/ bo.gao/freebu/, which was further disseminated [10].
}

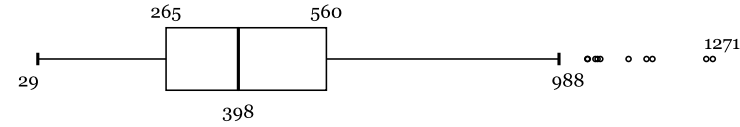

Fig. 1. Box plot for the number of friends per user. The minimum, 1st quartile, median, 3rd quartile and maximum are 29, 265, 398, 560 and 988 respectively. The 10 outliers are represented by circles.

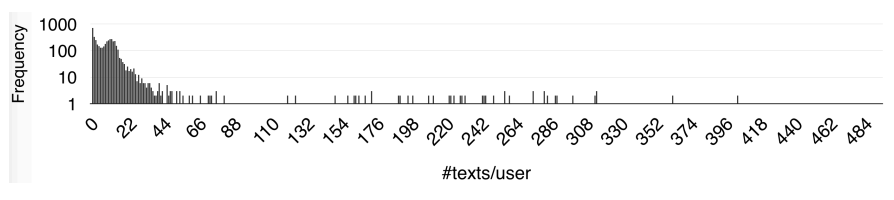

Fig. 2. Histogram of \#texts/user frequencies in chats

TABLE I. DATA SUMMARY OF chats AND posts

\begin{tabular}{lrrrrr}
\hline User Set & \#users & \multirow{2}{*}{ \#texts } & $\begin{array}{r}\text { \#texts } \\
\text { /user }\end{array}$ & $\begin{array}{r}\text { \#words } \\
\text { /text }\end{array}$ & $\begin{array}{r}\text { \#chars } \\
\text { /text }\end{array}$ \\
\hline chats & 4,480 & 84,751 & $10(11)$ & $6(9)$ & $23(35)$ \\
posts & 281,915 & $2,183,521$ & $2(3)$ & $6(9)$ & $29(41)$ \\
\hline
\end{tabular}

text. Shapiro-Wilk tests [12] on both the original values and log-scaled values of \#texts/user, \#words/text, and \#chars/text show that the respective distributions are significantly nonnormal $(p<.001)$. Indeed, we would expect exponential distributions here, such as Figure 2. The median and IQR (Inter-Quartile Range) values (IQR values in brackets) of \#texts/user, \#words/text and \#chars/text are summarized in Table I. We also see that the texts that people typed in chats and posts are short.

Language Identification: In order to automatically detect the sentiments of the texts, we first need to sort the texts based on the languages in which they were written. However, language identification is non-trivial because of the corpus' large size, the many users from different countries and the short lengths of the texts. Lui and Baldwin [13] selected and compared eight language identification systems on labeled Twitter texts. They showed that an equal-weight voting over three systems consistently outperforms any individual system. These systems are: LangID [14], LangDetect [15] and CLD2. ${ }^{3} \mathrm{We}$ adopt this method to identify the languages of the sentences in the corpus. HTML tags and URLs were removed beforehand. The results are summarized in Table II. In total, 70,389 texts in 48 languages $(83.1 \%$ of the original texts) from chats, and $1,890,476$ texts in 66 languages $(86.6 \%$ of the original texts) from posts, were identified. The languages of most texts in both chats and posts are Dutch, English, Spanish, German, French and Italian, as shown in Table II. The unidentified texts are usually very short phrases that are abbreviations, internet slang, (intentional) typos, emoticons and exclamation marks, such as “-_-Il”, “:)”, “STUDYYYYY!!!”, "Imao!”, or that occur in multiple languages such as "hehe","amen". Eventually, we analyzed $74.1 \%$ of the chats and $78.7 \%$ of the posts in 11 languages ${ }^{4}$.

\footnotetext{
${ }^{3}$ https://code.google.com/p/cld2/

${ }^{4}$ namely English, Dutch, French, German, Italian, Polish, Portuguese, Russian, Spanish, Swedish and Turkish
} 
2015 IEEE/ACM International Conference on Advances in Social Networks Analysis and Mining

TABLE II. DATA SUMmary For MaJor LANGUages

\begin{tabular}{rrr|rr}
\hline & \multicolumn{2}{c}{ chats } & \multicolumn{2}{c}{ posts } \\
Languages & \#texts & \#users & \#texts & \#users \\
\hline Dutch (nl) & 42,607 & 3,268 & 400,349 & 73,497 \\
English (en) & 10,977 & 1,894 & 635,997 & 117,521 \\
Spanish (es) & 1,835 & 347 & 247,358 & 42,672 \\
German (de) & 4,162 & 851 & 65,978 & 18,254 \\
French (fr) & 1,086 & 425 & 38,952 & 10,745 \\
Italian (it) & 867 & 377 & 180,211 & 32,415 \\
\hline
\end{tabular}

TABLE III. DATA SUMmary For PRIVACy LEVELS

\begin{tabular}{rrr|rrr}
\hline \multicolumn{2}{c}{ chats } & \multicolumn{2}{c}{ posts } \\
\#participants & \#texts & \#users & Privacy Setting & \#texts & \#users \\
\hline 2 & 53,983 & 3,249 & public & 362,038 & 71,665 \\
{$[3,4]$} & 4,054 & 572 & FoF & 67,151 & 13,737 \\
{$[5,6]$} & 1,625 & 393 & friends & $1,147,141$ & 177,350 \\
{$[7,10]$} & 1,698 & 529 & custom & 144,990 & 28,860 \\
{$[11,20]$} & 1,130 & 480 & & & \\
{$[21,64]$} & 329 & 235 & & & \\
\hline
\end{tabular}

TABLE IV. DATA SUMMARY FOR AGE

\begin{tabular}{rrr|rr}
\hline & \multicolumn{2}{c}{ chats } & \multicolumn{2}{c}{ posts } \\
Age & \#texts & \#users & \#texts & \#users \\
\hline$[13,16]$ & 544 & 59 & 1,684 & 94 \\
{$[17,20]$} & 15,516 & 754 & 65,676 & 1,776 \\
{$[21,24]$} & 15,627 & 816 & 236,128 & 4,167 \\
{$[25,28]$} & 7,696 & 480 & 167,839 & 2,454 \\
{$[29,32]$} & 2,744 & 208 & 79,763 & 984 \\
{$[33,36]$} & 1,405 & 99 & 36,088 & 399 \\
{$[37,40]$} & 274 & 26 & 13,235 & 174 \\
{$[41,50]$} & 419 & 54 & 24,629 & 303 \\
{$[51,60]$} & 175 & 30 & 9,484 & 144 \\
{$[61,80]$} & 129 & 8 & 989 & 37 \\
\hline
\end{tabular}

TABLE V. DATA SUmmary FOR RELATIONShip Status

\begin{tabular}{rrr|rr}
\hline Relation. & \multicolumn{2}{c}{ chats } & \multicolumn{2}{c}{ posts } \\
Status & \#texts & \#users & \#texts & \#users \\
\hline married & 626 & 36 & 81,029 & 856 \\
in a relationship & 4,673 & 158 & 205,004 & 2,462 \\
single & 2,818 & 190 & 196,107 & 2,089 \\
\hline
\end{tabular}

Attribute Selection / Construction: Each newsfeed post or chat record, with its comments, has an audience range, namely the set of (Facebook) users who can see the text. The texts in a chat are only visible to the chat participants. We can differentiate levels of privacy by the number of chat participants. The visibility of a text in a post is defined by its privacy setting, with four levels: public, friends of friends (FoF), friends and custom. The data statistics are summarized in Table III. For profile attributes, we have "age" and "relationship status", as shown in Table IV and V respectively. We chose the age groups similarly to [6]. Moreover, since it is unlikely for people older than 80 years old to use Facebook, we assume this data to be untrustworthy and exclude the corresponding users from our analysis. Also, we find that $99.9 \%$ of the users do not specify their "religion", "political-view" and "interested-in" features that are available in Facebook profiles.

Sentiment Analysis: We use SentiStrength [16] to produce the texts' sentiment scores. It is a lexicon-based system that detects polarized sentiment strengths of short informal texts. It takes into account both terms and other language features such as booster words, negation, emoticons, etc. Thelwall et al. [16] show that SentiStrength outperforms other common machine-learning algorithms. Abbasi et al. [17] further show
TABLE VI. Summary of Sentiment Strength Scores

\begin{tabular}{rrr|rr}
\hline & \multicolumn{2}{c}{ Chats } & \multicolumn{2}{c}{ Posts } \\
& Positive & Negative & Positive & Negative \\
\hline 1 & $43,305(68.9 \%)$ & $55,552(88.4 \%)$ & $1,074,092(62.3 \%)$ & $1,598,748(92.7 \%)$ \\
2 & $18,317(29.2 \%)$ & $6,664(10.6 \%)$ & $600,225(34.8 \%)$ & $102,543(6.0 \%)$ \\
3 & $1,117(1.8 \%)$ & $359(0.57 \%)$ & $44,910(2.6 \%)$ & $17,642(1.0 \%)$ \\
4 & $96(0.15 \%)$ & $263(0.42 \%)$ & $5,022(0.29 \%)$ & $5,767(0.33 \%)$ \\
5 & $7(0.01 \%)$ & $4(0.006 \%)$ & $572(0.03 \%)$ & $121(0.007 \%)$ \\
\hline
\end{tabular}

that SentiStrength is generally better than other similar tools on five benchmark datasets. Because the term weights and language rules of SentiStrength are previously defined, and no contextual texts are taken into account when predicting a text's sentiment, the positive and the negative scores of a text are generated independently and the positive/negative scores of different texts are generated independently. We run SentiStrength on the texts in chats and posts. The counts of texts with positive and negative sentiment are summarized in Table VI. Notice that most chats and posts are neutral (value \pm 1 ), and negative sentiment occurs less often than positve.

\section{Single-Attribute Sentiment Differences - HYPOTHESIS TESTING}

In this section we test the sentiment differences according to $R Q 1-R Q 3$. Because the sentiment scores are highly skewed, we use the non-parametric Mann-Whitney test [18] for two independent groups, and Kruskal Wallis test [19] for $>2$ independent groups. We report significant results ${ }^{5}$ with twotailed $p<.01$, with post-hoc tests Bonferroni-corrected [20]. We exclude the texts with unspecified age or relationship status, and merge the [37,50] and [51,80] age groups in chats to account for larger group size. We test both positive $\left(s^{+} \in[1,5]\right)$ and negative $\left(s^{-} \in[-5,-1]\right)$ sentiment differences. Also, when needed, we test sentiment expressiveness $\left(s^{+}-s^{-}\right)$differences. We will use the notation $\boldsymbol{G}_{A} \Phi \boldsymbol{G}_{B}$ with $\mathbb{\$} \in\{>,<, \approx\}$ to denote that group $\boldsymbol{G}_{A}$ is more, less than or similar to $G_{B}$ in terms of the absolute value of positive or negative sentiment, or expressiveness. Note that $\boldsymbol{G}_{A}>\boldsymbol{G}_{B}$ and $\boldsymbol{G}_{B} \approx \boldsymbol{G}_{C}$ does not automatically imply that $\boldsymbol{G}_{A}>\boldsymbol{G}_{C}$.

RQ1 (Privacy Level): Tests show that the private chats and public posts differ significantly in positive sentiment $(U=$ $\left.5.1 \times 10^{10}\right)$, negative sentiment $\left(U=5.2 \times 10^{10}\right)$ and expressiveness $\left(U=5.2 \times 10^{10}\right)$. More specifically, the texts in posts are more positive and expressive than those in chats. The texts in chats are more negative than those in posts. This indicates that people tend to express more positive sentiment in posts shared with a broad audience, whereas they feel more free to express less positive, and also less extreme sentiments in chats that are exchanged within a private circle of participants. This partially confirms our hypothesis in $R Q 1$ that there is indeed a general pattern that people are more positive and less negative in public than in private on Facebook. Within chats, there is a difference between the groups of different privacy levels in positive sentiment $\left(\chi^{2}(5)=29.0\right)$, and negative sentiment $\left(\chi^{2}(5)=83.5\right)$. The conversations involving [11-20] participants are both more positive and negative than those involving 2 participants. It coincides with the general pattern

\footnotetext{
${ }^{5}$ Due to the limited scope of this paper, we summarize and selectively report the results of the post-hoc pairwise tests in Section IV. A complete report can be found at http://goo.gl/R5k5iQ.
} 
2015 IEEE/ACM International Conference on Advances in Social Networks Analysis and Mining

TABLE VII. AgE GRoup EXPRESSIVENESS IN ChATS

\begin{tabular}{l}
\hline$[13,16]>[21,24]>[25,28],[29,32],[37,50],[51,80]$ \\
\hline$[17,20]>[33,36]>[21,24]>[25,28],[29,32]$ \\
\hline
\end{tabular}

that the texts are more sentimentally expressive in a more public setting. In posts, there is a difference in positive $\left(\chi^{2}(3)\right.$ $=840.6)$ and negative $\left(\chi^{2}(3)=130.1\right)$ sentiments between privacy levels: the $F o F$ (friends of friends) texts are both more positive and negative than the texts with other settings. The texts with the friends and custom settings are more positive than the public texts. We can see that the texts with a "fairly public" setting (namely $F o F$ ) are more expressive than others, but the sentiments of the public texts are generally reserved.

RQ2 (Age): In chats and posts, there is a difference between age groups in positive sentiment $\left(\chi_{\text {chats }}^{2}(7)=151.7\right.$, $\left.\chi_{\text {posts }}^{2}(9)=4,998.3\right)$, negative sentiment $\left(\chi_{\text {chats }}^{2}(7)=123.1\right.$, $\left.\chi_{\text {posts }}^{2}(9)=109.7\right)$ and expressiveness $\left(\chi_{\text {chats }}^{2}(7)=99.6\right.$, $\left.\chi_{\text {posts }}^{2}(9)=4,403.0\right)$. Post-hoc analysis reveals that younger people are generally more sentimentally expressive. The $[17,20]$ group is also more negative than older age groups in posts, which supports the hypothesis in $R Q 3$ that negative sentiment declines after the early 20 s, but we do not see an increase of negative sentiment in the mid-age range ([33,59]). Interestingly, we see the opposite in chats: the $[17,20]$ group is less negative than $[21,50]$. The late teen group seems to behave differently from older people in terms of negative sentiment expression. Younger people are generally more positive, which does not support the hypothesis that there is an increase of positivity after 50 years old.

RQ3 (Relationship Status): Tests show that there is also a difference between relationship-status groups, in positive sentiment $\left(\chi_{\text {chats }}^{2}(2)=66.7, \chi_{\text {posts }}^{2}(2)=2,642.4\right)$ and negative sentiment $\left(\chi_{\text {chats }}^{2}(2)=66.7, \chi_{\text {posts }}^{2}(2)=303.0\right)$. More specifically, in both chats and posts, the texts from single users express more positive sentiment than those from married users. Also, the posts from single users express more negative sentiment than those from married users. It shows a contrast with $R Q 3(a)$ that single users actually express themselves more positively than married users. For $R Q 3(b)$, in chats, single users express less negatively than users in a relationship. In posts, we find a stronger confirmation that users in $a$ relationship express both less positive and more negative sentiment than single users. For $R Q 3(c)$, users in a relationship have more positive chats and posts than those from married users. The users in a relationship also have more negative posts than the married users. These findings also show that married users are more neutral regarding online sentiment expression.

\section{Multi-Attribute Sentiment Differences - HYPOTHESIS EXPLORATION (RQ4)}

So far, we have analyzed the interplay between the groups of users defined by single attribute values and corresponding sentiments. It is relatively straightforward to apply statistical tests in such scenarios. However, oftentimes we need to look into the behaviour of user groups with combined attributes. For example, we find that the users with married relationship status tend to be less positive than the users with other statuses, but does this hold for both genders, different ages and so on? The

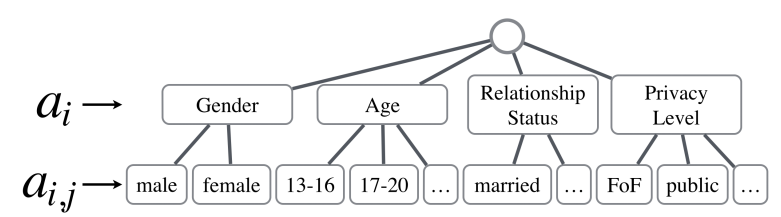

Fig. 3. Illustration of Hierarchy of Attribute Types and Values

solution to this problem falls under the paradigm of SubgroupDiscovery [21]. Recent advances [9] in the field have enabled fast discovery of "quality" subgroups with high diversity and low redundancy. However, the extracted subgroups are ranked according to certain quality measures, for instance, the mean difference between a subgroup and the entire population. This could complicate the interpretation of the results in our case, because we want to compare subgroups. To this end, we develop two algorithms to extract such comparisons, which not only reveal interesting attribute combinations that provide a more fine-grained insight into the relationships between attributes and sentiments, but also offer potential sociological hypotheses for future study.

\section{A. Vertical Comparisons}

Consider a hierarchy $A$ of attribute types (labeled $a_{i}$ ) and values (labeled $\left.a_{i, j}\right), i, j \in \mathbb{N}$, as shown in Figure 3. Namely, $A=\left\{\left(a_{i}, A_{i}\right)\right\}, A_{i}=\left\{a_{i, j}\right\}$. We define the complement of an attribute value $a_{i, k}$ within $A_{i}$ as $A_{i,(k)}^{\prime}=\left\{a_{i, j} \mid j \neq\right.$ $\left.k, a_{i, j} \in A_{i}\right\}$. Similarly, the complement of an attribute $a_{i}$ in the scope of $A$ is defined as $A_{(i)}^{\prime}=\left\{A_{j} \mid i \neq j,\left(a_{j}, A_{j}\right) \in A\right\}$. Note that the algorithms (Section V-A and V-B) can be straightforwardly extended to accommodate a hierarchy with more levels of attribute values. For example, the age interval can be coarse initially, but divided into finer intervals at deeper levels. Consider a subgroup $\boldsymbol{G}$ as a set of attribute values $\left(a_{i, j}\right)$ where each corresponding attribute type $\left(a_{i}\right)$ appears zero or one time. For example, a subgroup can be the females within 21-24 years old, namely \{female, 21-24\}. Let $\mathcal{G}=\left\{\boldsymbol{G}_{i}\right\}$ be the set of these subgroups. We use $\mathrm{m} \in M$ with $M=\{$ pos, neg,express $\}$ to denote a chosen measure of positive sentiment, negative sentiment and expressiveness. We use the sign $\Phi \in\{>,<, \approx\}$, as defined in Section IV, to describe the relationship between two subgroups, with the measure $\mathrm{m} \in M$, according to the statistical test $\mathrm{t}$ and the significance level $\alpha$. Let $t\left(\boldsymbol{G}_{A}, \boldsymbol{G}_{B}, \mathrm{~m}\right)$ be the test that returns the sign $\$$ and the two-tailed p-value $p$, on subgroups $\boldsymbol{G}_{A}$ and $\boldsymbol{G}_{B}$ with the measure $\mathrm{m}$.

The algorithm for finding "vertical comparisons" of subgroups is detailed below. We use the set of "base comparisons", $\mathcal{C}_{\text {base }}$, to store the comparisons between an attribute value and its complement within the same attribute, namely $\mathcal{C}_{\text {base }}=\left\{\left(\left\{a_{i, k}\right\}, A_{i,(k)}, \Phi, \mathrm{m}, p\right)\right\}$, and the set of comparisons $\mathcal{C}$ to store the comparisons between a target subgroup $G$ (with $|\boldsymbol{G}|>1$ ) and its "counterpart" $S$ (with $|\boldsymbol{G}|=|\boldsymbol{G} \cup S|+1$ ). We then have $\mathcal{C}=\{(\boldsymbol{G}, S, \Phi, \mathrm{m}, p)\}$. A depth-first search progressively accounts for subgroups with higher orders of attribute-value combinations. The significance level $\alpha$ serves as the pruning threshold that stops the search at a branch if the corresponding test's p-value is larger than $\alpha$ (Line 19-22). 
TABLE VIII. EXAMPLES OF VERTICAL COMPARISONS

\begin{tabular}{cl|l}
\hline data, $\mathrm{m}$ & comparison & base \\
\hline chats, pos & $\{$ female, $21-24\}<\{$ female, $\neg(21-24)\}$ & $\{21-24\} \approx\{\neg(21-24)\}$ \\
posts, pos & $\{17-20$, relation. $\}<\{17-20, \neg$ (relation. $)\}$ & $\{$ relation. $\}>\{\neg$ (relation. $)\}$ \\
posts, neg & $\{25-28$, friends $\}>\{25-28, \neg$ (friends) $\}$ & $\{$ friends $\}<\{\neg$ (friends) $\}$ \\
\hline
\end{tabular}

Given $A, \mathrm{~m}$

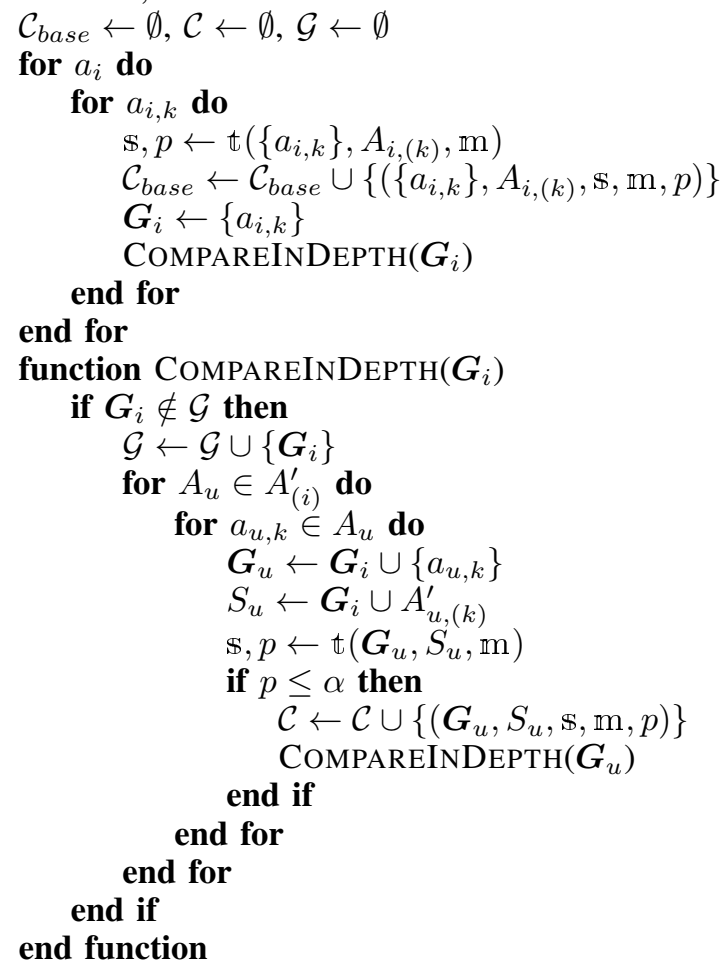

The algorithm returns two filled sets of comparisons $\mathcal{C}_{\text {base }}$ and $\mathcal{C}$. $\mathcal{C}_{\text {base }}$ contains the comparisons of single attribute values and their complements, informing us whether and how an attribute value is distinguished from the rest. $\mathcal{C}$ contains the comparisons of attribute combinations in different orders and their more general counterparts, informing us that by adding a specific attribute value, whether and how a combination is distinguishable from the rest. Table VIII shows the examples of vertical comparisons with the smallest p-values. For example, while there is no positive sentiment difference between the chats from the people of 21-24 years old and other ages, adding the "gender=female" attribute value reveals that, in contrast, females of 21-24 years old have less positive chats compared to other females. See in the 3rd example that, while the posts with the friends setting are generally less negative than those with other settings, the people of 25-28 years old express themselves more negatively in this setting.

\section{B. Horizontal Comparisons}

While the vertical comparisons help us see the effect of adding/removing one attribute value on sentiment distributions, it is also desirable to see how different values of the same attribute affect sentiment distributions under more complex conditions. For example, how do \{male, relation.\}, \{male, married $\},\{$ male, single $\}$ differ from each other? To this end, we modify the algorithm in Section V-A to extract horizontal comparisons, as shown below. Statistical tests are performed
TABLE IX. EXAMPLES OF HORIZONTAL COMPARISONS

\begin{tabular}{rl|l}
\hline data, m & comparison & base \\
\hline posts, express & $\{$ custom, $17-20\}<\{$ custom, 37-40\} & $\{17-20\}>\{37-40\}$ \\
posts, pos & $\{$ male, married $\}>\{$ male, single $\}$ & $\{$ married $\}<$ single $\}$ \\
posts, pos & $\{$ male, relation. $\}>\{$ male, single $\}$ & $\{$ relation. $\}<\{$ single $\}$ \\
posts, pos & $\{$ male, married $>\{$ male, relation. $\}$ & $\{$ married $\}<$ relation. $\}$ \\
\hline
\end{tabular}

on a set of subgroups corresponding to all the attribute values $a_{u, k}$ under an attribute $a_{u}$, conditioned on a previously given subgroup $\boldsymbol{G}_{i}$ (Line 15-23). Furthermore, let $\mathcal{G}^{\prime}=\left\{\boldsymbol{G}_{i}\right\}$ $\left(\left|\mathcal{G}^{\prime}\right| \geq 2\right)$ be a set of subgroups subject to post-hoc analysis, and $\mathbb{t}\left(\mathcal{G}^{\prime}, \mathrm{m}, \alpha\right)$ the function that performs the pairwise testing and returns a set of comparisons that are significant at $\alpha$ level. Similar to the algorithm in Section V-A, $\alpha$ serves as a threshold to remove the comparisons with large p-values.

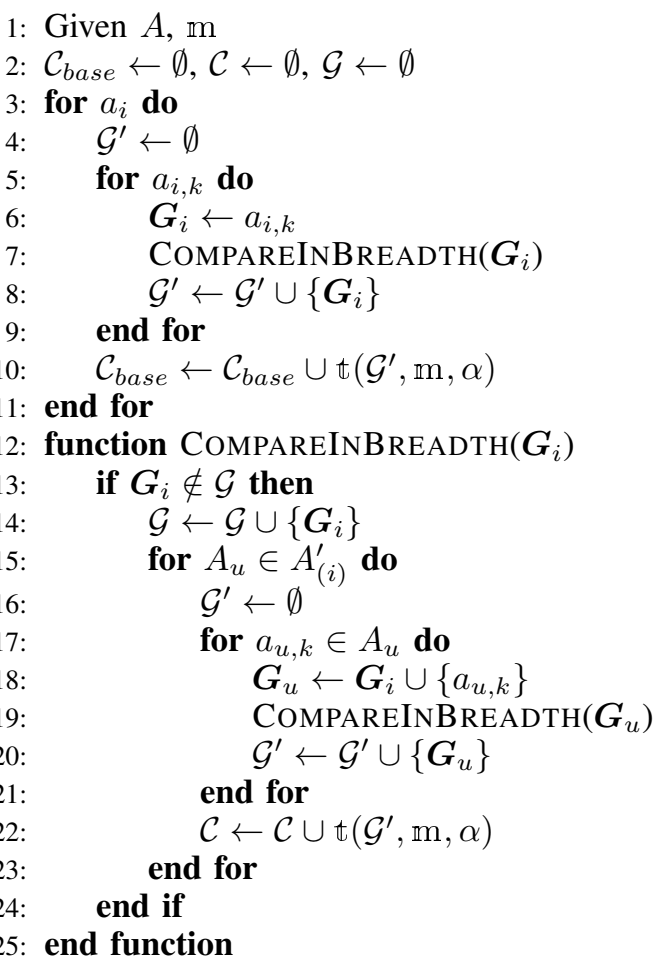

Table IX shows examples of horizontal comparisons with the smallest p-values. For example, from Section IV we know that younger people are more sentimentally expressive, as one base comparison $\{17-20\}>\{37-40\}$ shows. However, when the privacy setting is custom, the expressiveness reverses, suggesting that the $\{17-20\}$ group is not as expressive as they would be in a more public setting, and/or the $\{37-$ $40\}$ group expresses themselves more freely in a more private setting. Moreover, from Section IV we know that in posts, the positive sentiment differences in relationship status are: $\{$ single $\}>\{$ relation. $\}>\{$ married $\}$, but this pattern reverses when adding the "gender=male" attribute value, as shown in the table, providing us with a more differentiated view on the positive sentiment differences in relationship status.

\section{LimitATIONS AND OUTLOOK}

We apply statistical tests to identify differences between groups of sentiment scores, based on the assumption that each text's sentiment is independent of other texts' sentiments. This 
assumption has two limitations: first, the sentiments of the texts from the same user may be correlated; second, the sentiments of the texts from the same chat or post may be correlated as well. Also, as seen in Section III, the user sample in our dataset is biased. It consists of mostly young people from west European countries, particularly so for the users in chats, who are mostly Flemish students. Moreover, we only considered the users who have available profile features for demographical factors, which increases the bias. Furthermore, we used a tool (SentiStrength) to extract sentiment scores from the texts in multiple languages, which is bound to produce errors. Although it has been shown to be encouragingly accurate in relevant domains (Section II, III), it is yet to be investigated to which extent the inaccuracies may affect our results. We exclude the texts of which the language is unidentified. These texts include punctuations, emoticons and universal phrases, which account for a small proportion, but may still have an impact. Finally, it is inherently difficult and ambiguous to rate a given sentence's sentiment. Oftentimes, people use negative words to be humorous or sarcastic, which could be counted as "positive". Sentiments also heavily depend on their contexts. Future studies can utilize context-based multidimensional sentiment analysis (e.g. [22]).

\section{CONCLUSION}

In this paper, we take an interdisciplinary approach towards mining the patterns inherent to textual sentiments and metadata in online social networks. We investigate the sentiment differences across privacy levels and demographic factors and find that not only the "conventional" or "stereotypical" hypotheses on demographic groups' sentiment expression are challenged, but also, importantly, that there are more detailed "stories" to be explored. For example, we find that the "coming of age" $[17,20]$ group wrote less negative texts in chats than older age groups, which counters our hypothesis that lateteens have more negative texts. Furthermore, while most social data analysis focuses on publicly available texts, we see different sentiment expressions from users under different privacy settings. It reminds us that people naturally adjust their communication with others according to the size of the audience, among many other factors. Investigating these differences will improve our understanding of the data. For example, we find that the texts posted publicly are in general more positive than those posted privately, but the texts with a complete public setting are more reserved. Finally, using the subgroup-discovery paradigm, we present an approach with two algorithms that generalizes single-attribute testing, so as to provide more detailed insight into the relationships among different attributes, reveal interesting attribute-value combinations with distinct sentiments, and provide novel hypotheses for examination in future studies.

\section{ACKNOWLEDGMENT}

We thank Prof. Thelwall for his support with the SentiStrength tool. The research presented in this paper was supported by the Strategic Basic Research (SBO) Programme of the Flemish Agency for Innovation through Science and Technology (IWT) through the project SPION (grant number 100048), and from the organization Fund for Scientific Research for Flanders (FWO) through the project Data Mining for Privacy in Social Networks (grant number G068611N).

\section{REFERENCES}

[1] A. D. Kramer, J. E. Guillory, and J. T. Hancock, "Experimental evidence of massive-scale emotional contagion through social networks," Proceedings of the National Academy of Sciences, vol. 111, no. 24, pp. 8788-8790, 2014.

[2] A. Siganos, E. Vagenas-Nanos, and P. Verwijmeren, "Facebook's daily sentiment and international stock markets," Journal of Economic Behavior \& Organization, vol. 107, pp. 730-743, 2014.

[3] M. Thelwall, D. Wilkinson, and S. Uppal, "Data mining emotion in social network communication: Gender differences in MySpace," Journal of the American Society for Information Science and Technology, vol. 61, no. 1, pp. 190-199, 2010.

[4] J. J. Gross, J. M. Richards, and O. P. John, "Emotion regulation in everyday life," Emotion regulation in couples and families: Pathways to dysfunction and health, vol. 2006, pp. 13-35, 2006.

[5] J. J. Gross, L. L. Carstensen, M. Pasupathi, J. Tsai, C. Götestam Skorpen, and A. Y. Hsu, "Emotion and aging: experience, expression, and control." Psychology and aging, vol. 12, no. 4, p. 590, 1997.

[6] A. A. Stone, J. E. Schwartz, J. E. Broderick, and A. Deaton, "A snapshot of the age distribution of psychological well-being in the United States," Proceedings of the National Academy of Sciences, vol. 107, no. 22, pp. 9985-9990, 2010.

[7] S. C. Yap, I. Anusic, and R. E. Lucas, "Does personality moderate reaction and adaptation to major life events? Evidence from the british household panel survey," Journal of research in personality, vol. 46, no. 5, pp. 477-488, 2012.

[8] J. Taylor, Emotional experience and romantic relationship status in emerging adult college women and men. Colorado State University, 2009.

[9] M. van Leeuwen and A. Knobbe, "Diverse subgroup set discovery," Data Mining and Knowledge Discovery, vol. 25, no. 2, pp. 208-242, 2012.

[10] R. De Wolf, B. Gao, B. Berendt, and J. Pierson, "The promise of audience transparency: Exploring users' perceptions and behaviors towards visualizations of networked audiences on Facebook," Telematics and Informatics, vol. 32, no. 4, pp. 890-908, 2015.

[11] J. W. Tukey, "Box-and-whisker plots," Exploratory Data Analysis, pp. 39-43, 1977.

[12] S. S. Shapiro and M. B. Wilk, "An analysis of variance test for normality (complete samples)," Biometrika, pp. 591-611, 1965.

[13] T. B. Marco Lui, "Accurate language identification of Twitter messages," in Proceedings of the 5th Workshop on Language Analysis for Social Media (LASM)@EACL, 2014, pp. 17-25.

[14] M. Lui and T. Baldwin, "langid. py: An off-the-shelf language identification tool," in Proceedings of the ACL 2012 System Demonstrations. Association for Computational Linguistics, 2012, pp. 25-30.

[15] S. Nakatani, "Language Detection Library for Java," https://code.google. com/p/language-detection/, 2011.

[16] M. Thelwall, K. Buckley, G. Paltoglou, D. Cai, and A. Kappas, "Sentiment strength detection in short informal text," Journal of the American Society for Information Science and Technology, vol. 61, no. 12, pp. 2544-2558, 2010.

[17] A. Abbasi, A. Hassan, and M. Dhar, "Benchmarking Twitter sentiment analysis tools," in The International Conference on Language Resources and Evaluation, 2014, pp. 823-829.

[18] H. B. Mann and D. R. Whitney, "On a test of whether one of two random variables is stochastically larger than the other," The annals of mathematical statistics, pp. 50-60, 1947.

[19] W. H. Kruskal and W. A. Wallis, "Use of ranks in one-criterion variance analysis," Journal of the American statistical Association, vol. 47, no. 260, pp. 583-621, 1952.

[20] O. J. Dunn, "Estimation of the medians for dependent variables," The Annals of Mathematical Statistics, pp. 192-197, 1959.

[21] M. Atzmueller and F. Puppe, "SD-Map-A fast algorithm for exhaustive subgroup discovery," in Knowledge Discovery in Databases: PKDD 2006. Springer, 2006, pp. 6-17.

[22] K. R. Scherer, "What are emotions? And how can they be measured?" Social science information, vol. 44, no. 4, pp. 695-729, 2005. 